\title{
MASTITIS REVEALING EOSINOPHILIC GRANULOMATOSIS WITH POLYANGIITIS: A CASE REPORT
}

Luiz Felipe Dipe Prates Miranda ${ }^{1, \star}$, Maria Carolina Fonseca Coelho ${ }^{1}$, Rafaela Vieiralves João Izzo Pinto ${ }^{1}$, Kátia Cristina Carvalho de Melo Matos ${ }^{1}$, Débora Rocha de Moura Rodrigues de Aguiar ${ }^{1}$

1.Hospital Federal de Bonsucesso, Rio de Janeiro (RJ), Brazil.

*Corresponding author: luizfelipedipe@gmail.com

\section{BACKGROUND}

Eosinophilic granulomatosis with polyangiitis (EGPA), in the past called Churg-Strauss syndrome, is a systemic necrotizing vasculitis of small and medium-size vessels, characterized by asthma and blood eosinophilia. Eosinophilic granulomatosis with polyangiitis typically occurs in patients with preexisting asthma and involves the skin, lungs and peripheral nerves.

\section{CASE-REPORT}

A 36-year-old female presented a bilateral yellowish-green papillary discharge (Fig. 1). She reported pain, edema and redness in both breasts plus fever for over a month. Unlikely most common mastitis, this one would not get better with oral or parenteral antibiotics. She had had severe asthma with recurrence in adulthood and eight episodes of pneumonia in the last few years. Complementary exams showed 1054 (17\%) eosinophils in the blood cell count. An inflammatory infiltrate in series of thorax computed tomography (CT) scans. A paranasal sinus CT showing veiling of the frontal sinuses, anterior and posterior ethmoidal cells; sphenoid mucous thickening and nasal turbinate hypertrophy. A breast core biopsy pointing mastitis associated with vasculitis plus an intense eosinophilic infiltrate and an esophagus biopsy revealing a chronic esophagitis, which was rich in eosinophils. A negative antineutrophil cytoplasmic antibodies (ANCA) and presence of polyneuropathy confirmed in an electroneuromyography exam. The patient was diagnosed with EGPA syndrome, filling in all six criteria defined in 1990 by the American College of Rheumatology, starting treatment with glucocorticoids and cyclophosphamide reaching disease control. The authors declare that the patient was oriented and signed the written informed consent form.

\section{CONCLUSION}

What draws attention in this case is the eosinophilic mastitis, which was the main symptom that revealed the EGPA's syndrome. Until the closure of this clinical case there has been no other case report like that in the literature. 